\title{
Periodontal regeneration of an intrabony osseous defect with combination of platelet rich fibrin and bovine derived demineralized bone matrix: A case report
}

\author{
Dr. K. Malathi, M.D.S ${ }^{1}$, Dr. A.Muthukumaraswamy, M.D.S. ${ }^{2}$, \\ Dr. Shruti Beri, B.D.S., (M.D.S.) ${ }^{3}$ \\ 1, 2,3 (Department of Periodontics, Tamil Nadu Government Dental College and Hospital, Chennai, India)
}

\begin{abstract}
Background: The ultimate goal of periodontal therapy is regeneration of tissues destroyed by periodontal disease. Previously, various bone graft materials have been tried for management of osseous defects resulting from the disease process. However, the trend has now shifted towards application of endogenous regenerative therapy. The recent introduction of Choukroun's Platelet Rich Fibrin (PRF), has revolutionalized the field of dentistry. PRF, a rich source of autologous growth factors and cytokines, is an upcoming therapeutic approach in the management of periodontal osseous defects. Combination of autologous $P R F$ and the otherwise commercially available bone grafts holds a promising potential of enhanced bone regeneration.

Aim: The aim of this case report is to present the management of an intrabony osseous defect with combination of Platelet Rich Fibrin \& Bovine derived demineralised bone matrix with clinical and radiographic evaluations for a 6 months follow up period.

Material and Methods: A 40 year old female patient presented with the chief complaint of pain in relation to \#13. Clinical evaluation revealed probing pocket depth (PPD) of $10 \mathrm{~mm}$ and clinical attachment level $(C A L)$ of $11 \mathrm{~mm}$ distal to \#13. Digital Radiographic analysis revealed angular bone loss in the region. Surgical therapy involved management by means of open flap debridement and placement of combination of autologous PRF and demineralized bone matrix in the combined wall defect. PRF was also used as membrane to secure the defect.

Result: Post-operative evaluations revealed significant reduction in PPD and CAL gain at the end of 6 months. Also radiographic evidence of bone formation was observed as early as 3 months with almost complete fill by 6 months post-operatively.

Conclusion: The combination therapy using platelet rich fibrin with demineralised bone matrix poses to be a rapid, effective and promising grafting modality for the management of intrabony osseous defects.
\end{abstract}

Keywords: Demineralized bone matrix, Intrabony Defect, Platelet concentrates, Platelet Rich fibrin

\section{Introduction}

Periodontitis is an inflammatory disease characterized by destruction of alveolar bone, root cementum, periodontal ligament and gingiva as a response to insults elicited by microbial accumulations on tooth surfaces. ${ }^{1}$ These responses tend to result in variety of intraosseous defects of various architectures.

Periodontal therapy is performed with the primary objectives of gaining access to the diseased sites, achieving reduction in pocket depth, arresting further disease progression and finally restoring the periodontal tissues lost due to disease process. The ultimate aim is to achieve periodontal regeneration via new attachment formation. Regeneration has been defined as the reproduction or reconstitution of a lost or injured part to restore the architecture and function of the periodontium. ${ }^{2}$

Conventional open flap debridement offers only limited potential towards recovering the lost periodontal structures. ${ }^{3}$ Various grafting modalities have been used for periodontal tissue regeneration such as autogenous ${ }^{4,5}$ and allogenic bone grafts ${ }^{6}$ but none has been established as a gold standard in the treatment of intrabony defects, given their own associated limitations. ${ }^{7}$

Recently, the attention has shifted towards use of growth factors to regulate various cell-stromal interactions involved in periodontal regeneration. These growth factors are the vital biologic mediators that can regulate the proliferation, chemotaxis and differentiation of the locally derived progenitor cells in the defect site. $^{8}$

Platelets apart from their role in hemostasis, have been reported to possess regenerative potential as their alpha granules are rich sources of various vital growth factors. ${ }^{9}$ Technological advancement has led to development of concentrated platelets, by means of centrifugation, popularly known as Platelet concentrates.

Platelet rich fibrin (PRF) the second and the latest generation of platelet concentrate is an emerging therapeutic modality in the fields of medicine and dentistry. It's completely autogenous nature, with no artificial 
biochemical agents involved, makes PRF a safe and inexpensive treatment modality. ${ }^{10}$ The physiologic fibrin matrix of PRF, obtained as the result of slow polymerization, has the ability to hold various growth factors and cytokines and release them at the wound site for a prolonged time period. ${ }^{11}$ The Leukocytes and key immune cytokines like IL 1 $\beta$, IL 6, IL 4 and TNF $\alpha$ trapped in PRF give it the anti-infectious effect and lets PRF act as an immune regulation node. ${ }^{12}$ All these properties makes Platelet Rich Fibrin a unique entity in itself.

The application of PRF in fields of plastic surgery ${ }^{13}$, oral and maxillofacial surgery ${ }^{14}$ and implant surgery ${ }^{15}$ has demonstrated successful and rapid results in terms of bone regeneration. The field of Periodontal therapy, although, has just begun to explore the vast benefits of PRF. Management of furcation defects, ${ }^{16}$ multiple gingival recessions ${ }^{17}$ and intrabony defects ${ }^{18}$ with the help of PRF have shown promising results.

Demineralized bone matrix (DMBM) is a sterile bio-resorbable bovine derived xenograft composed of type I collagen which has demonstrated positive results in management of intrabony defects. ${ }^{19}$ Given the unique properties of autologous PRF and already demonstrated regenerative capacity of commercially available bone graft, application of a combination approach was attempted for the assessment of their additional benefits to the healing mechanism and periodontal regeneration. Thus, we present here a six month follow-up report of an intrabony defect, treated by means of combination of autologous PRF with bovine derived demineralised bone matrix, and assessed clinically and radiographically.

\subsection{Chief Complaint and initial examination}

\section{Case Report :}

A 40-year-old Indian female reported to the Department of Periodontics, Tamil Nadu Government Dental College and Hospital, Chennai, India with the chief complaint of mild pain in relation to upper right front tooth for past 1 week, with a recent episode of swelling \& pus discharge that subsided after medication. Patient also gave history of similar episodes 3-4 times in past 1 year. She did not give any relevant medical history and was reported to be in systemically healthy condition. There was no history of dental trauma or injurious habit reported by the patient.

On intraoral examination, distobuccal aspect of tooth \#13 revealed probing pocket depth (PPD) of $10 \mathrm{~mm}$, with clinical attachment level (CAL) of $11 \mathrm{~mm}$. No mobility was detected in relation to13 and fremitus was found to be mildly positive (Figure 1). Although bleeding on probing and pus exudation was present but no signs of swelling were noticed. A digital periapical radiograph taken using long cone technique, revealed presence of interdental angular bone loss distal to tooth \#13. (Figure 2)

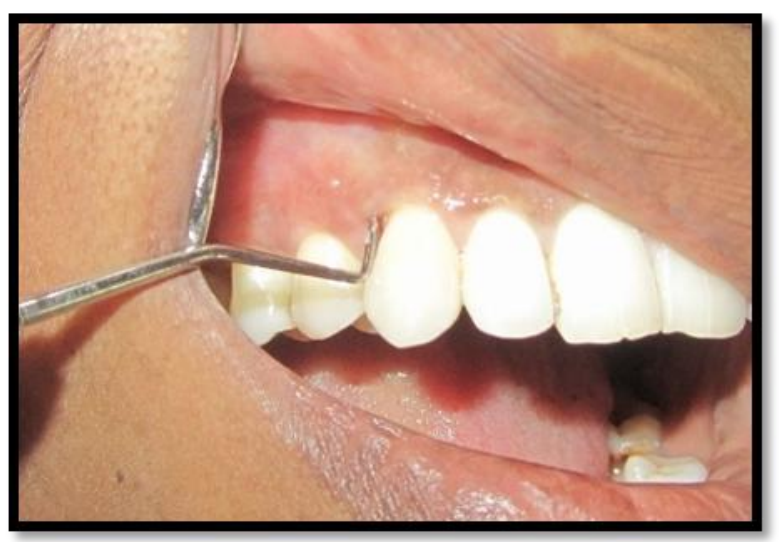

Figure 1 Pre-operative clinical view (Distal \#13)

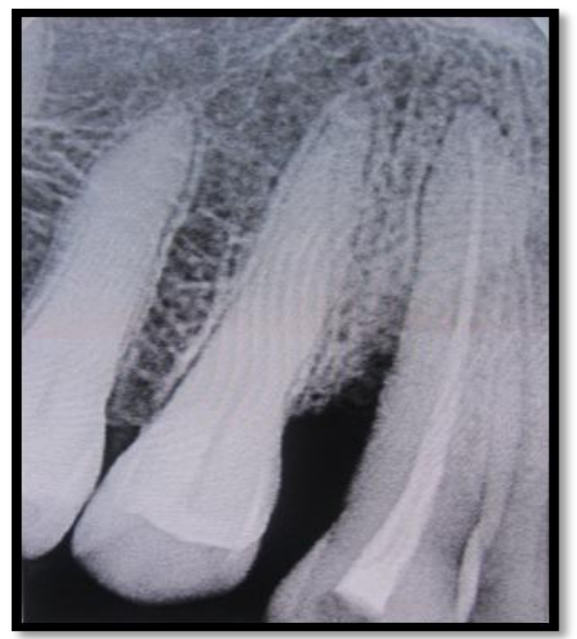

Figure 2 : Pre-operative radiographic view

\subsection{Treatment plan included:}

1. Administration of antibiotics and analgesics for 5 days

2. Routine blood investigations

3. Oral hygiene instructions and motivation of the patient in performing effective oral hygiene measures.

4. Non-surgical periodontal therapy by means of ultrasonic instruments.

5. After a period of 1 week, scaling and root planning, using curettes

6. Endodontic evaluation for vitality of tooth and Root canal treatment

7. Clinical re-evaluation after 4 weeks

8. Surgical periodontal therapy

9. Supportive periodontal maintenance 
10. Clinical re-evaluation at the end of 6 months

11. Digital radiographic re-evaluations at the end of 3 months and 6 months

After initial patient care, oral prophylaxis was performed and patient was evaluated for acceptable oral hygiene maintenance. Routine blood investigations revealed patient's platelet count $\left(3.51 \mathrm{lac} / \mathrm{mm}^{3}\right)$, Haemoglobin $(13.5 \mathrm{gm} / \mathrm{dl})$, Bleeding time $(2.5 \mathrm{~min})$, Clotting time $(4.5 \mathrm{~min})$ and random blood sugar $(124 \mathrm{mg} / \mathrm{dl})$ to be within normal limits. Endodontic therapy was performed given the non-vital response of the tooth and occlusal adjustments were done.

Re-evaluation after 4 weeks following non-surgical periodontal therapy revealed PPD and CAL to be still $9 \mathrm{~mm}$ and $10 \mathrm{~mm}$ respectively. Therefore, access flap surgery was planned.

\subsection{Surgical Procedure:}

Intra-oral antisepsis and extraoral antisepsis was performed with $0.2 \%$ chlorhexidine digluconate rinse and povidone iodine solution respectively. Following administration of local anaesthesia, buccal and palatal sulcular incisions were made and mucoperiosteal flaps were reflected involving the two adjacent teeth. A combined intabony defect (with three walls apically and two coronally) was thus revealed distal to \#13 (Figure 3a). Care was taken to preserve as much inter-proximal soft tissue as possible. Root planning and complete defect debridement was carried out. No osseous recontouring was performed. Freshly prepared PRF was obtained, made into small pieces and combined with demineralised bone matrix (osseograft ${ }^{\mathrm{TM}}$ ) in equal proportions $(1: 1 \mathrm{v} / \mathrm{v})^{20}$. The intrabony defect was filled with the mixture using light pressure till the defect walls (Figure 3b). And the coronal part (i.e. two walled defect) was covered with PRF membrane alone (Figure 3c). The mucoperiosteal flaps were repositioned and secured in place using 3-0 non-absorbable braided black silk surgical suture. Simple interrupted sutures were placed (Figure 3d) and the surgical area was protected and covered with periodontal dressing.

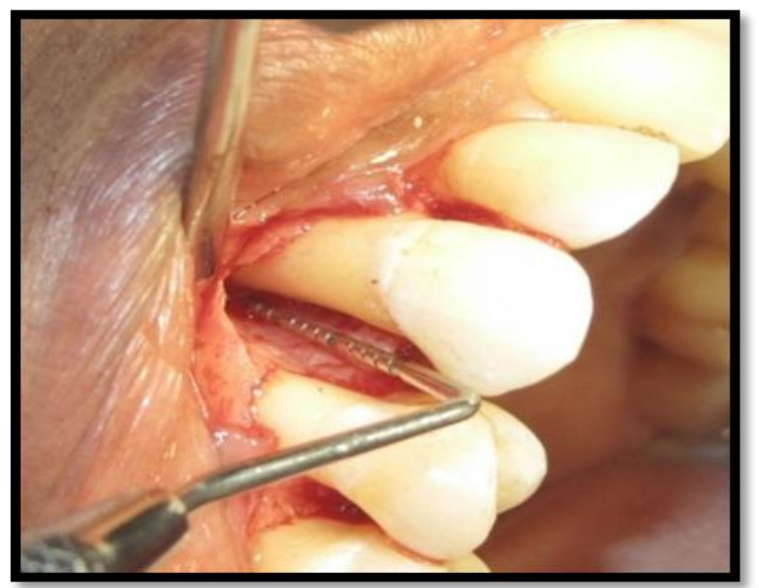

Figure 3(a): Combined wall defect: Intra operative view

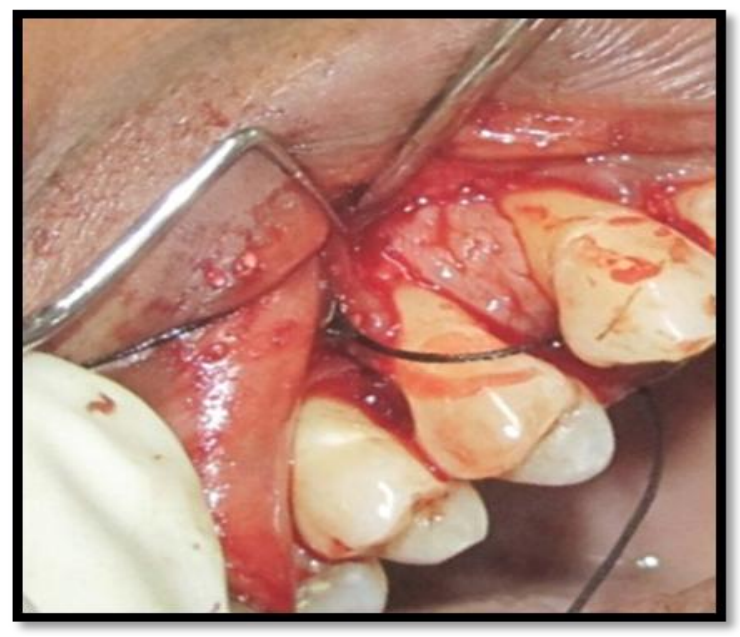

Figure 3 (c) PRF membrane used to cover the defect

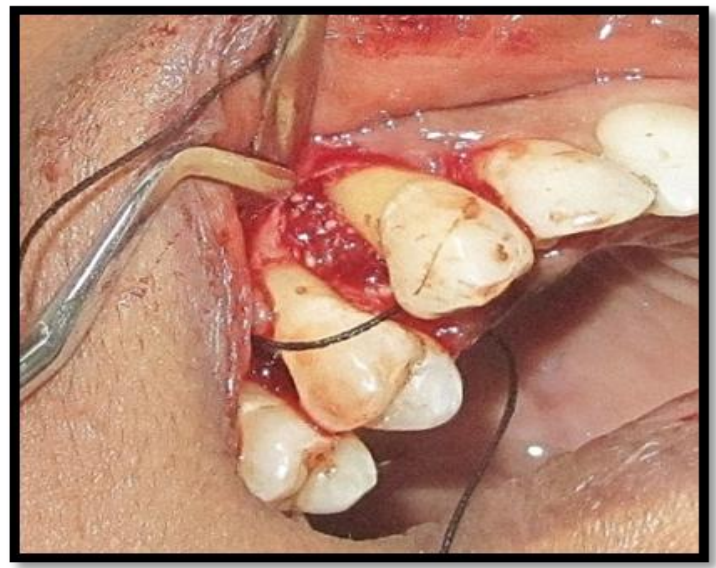

Figure 3 (b) PRF mixed with DMBM placed in defect

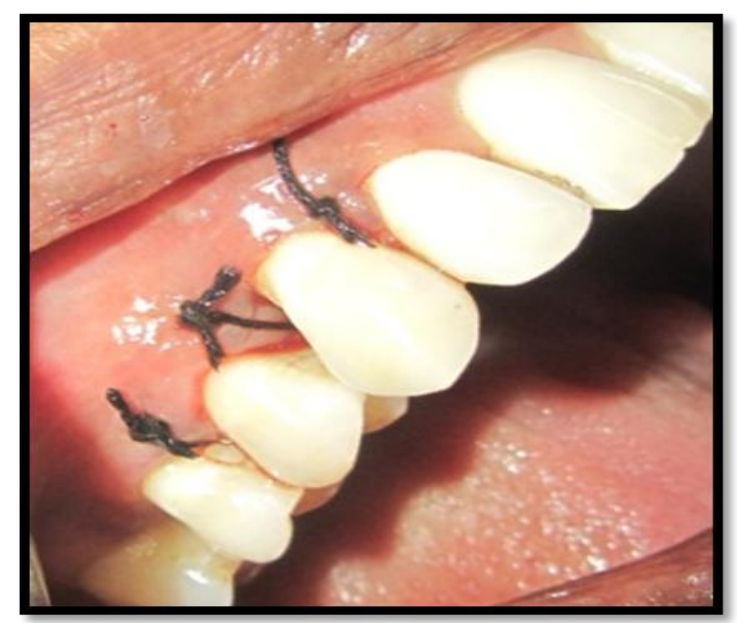

Figure 3(d) : After suturing 


\subsection{PRF Preparation:}

The PRF was prepared in accordance with the protocol developed by Choukroun et al ${ }^{10}$. Just prior to surgery, intravenous blood (from the antecubital vein) was collected in a 10-ml sterile glass tube without anticoagulant and immediately centrifuged in centrifugation machine at $3000 \mathrm{rpm}$ for 10 minutes. It resulted in separation of blood (Figure 4a) into a structured fibrin clot in the middle of the tube, just between the red corpuscles at the bottom and acellular plasma (Platelet-poor plasma) at the top. ${ }^{10}$ PPP was discarded, PRF was easily separated from red corpuscles base using sterile tweezers and scissors. The junction of PRF to RBC layer was preserved (Figure $4 \mathrm{~b}$ ), as this region is supposed to be richest in all the growth factors. ${ }^{11}$ The prepared PRF was immediately used as per the studies reported by $\boldsymbol{S} \boldsymbol{u} \boldsymbol{e t} \boldsymbol{a l}{ }^{22}$ Half of the PRF was made in pieces and mixed with equal amount of bone graft to fill the defect. Other half was used as a membrane to cover the defect as a third wall.

\subsection{Postoperative Care:}

The Suitable antibiotics and analgesics were prescribed, along with chlorhexidine digluconate rinses $(0.2 \%)$ twice daily for 2 weeks. Periodontal dressing and sutures were removed after 10 days. Saline irrigation was done and patient was instructed for gentle brushing with a soft toothbrush. Re-evaluation was done weekly for up to 1 month after surgery and then at 3 and 6 months. No subgingival instrumentation was attempted at any of these appointments.

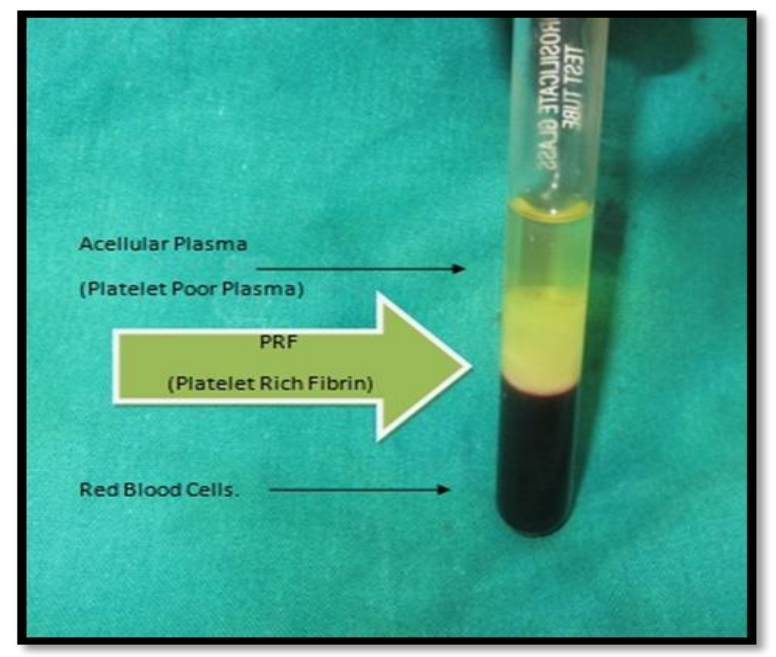

Figure 4. a) Patients blood immediately after centrifugation

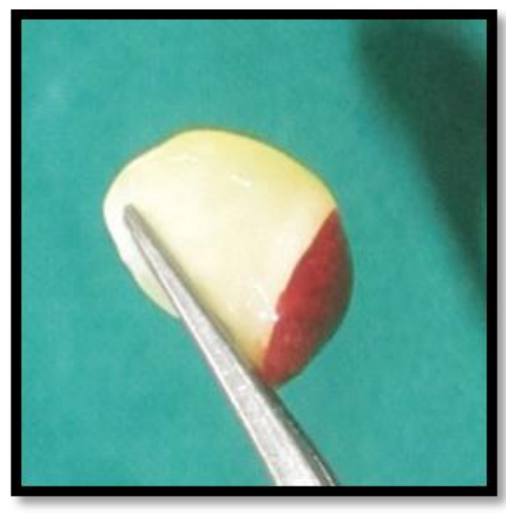

Figure 4(b) PRF gel

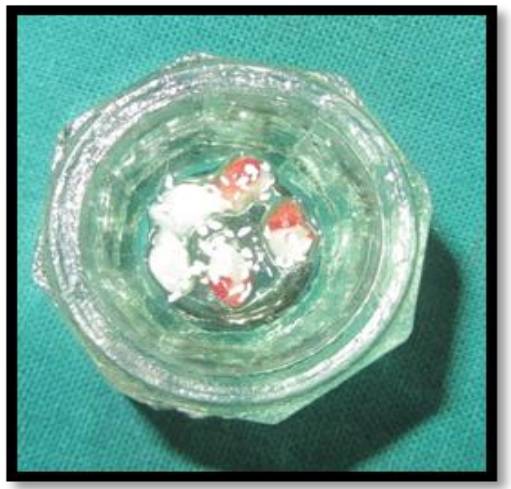

Figure 4 (c) PRF mixed with DMBM granules

\subsection{Results}

The patient showed good compliance and satisfactory oral hygiene maintenance during the course of observation period. The healing was uneventful, without any signs of infections and complications, indicating biocompatibility of both grafting modalities.

Clinical re-evaluation at 6 months after the periodontal surgery revealed PPD of $3 \mathrm{~mm}$ and CAL of 4 $\mathrm{mm}$ with no signs of bleeding on probing (Figure 5). Digital radiographic re-evaluations were performed at 
3 months and 6 months post-operatively. The radiographic analysis of the defect revealed significant bone formation at the end of 3 months with additional crestal bone formation followed by almost complete bone fill by 6 months. (Figure 6a, 6b)

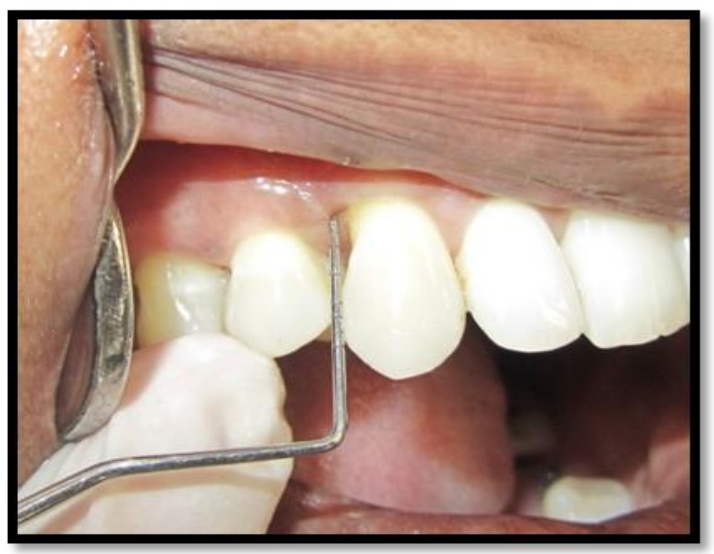

Figure 5: Post-operative Clinical view

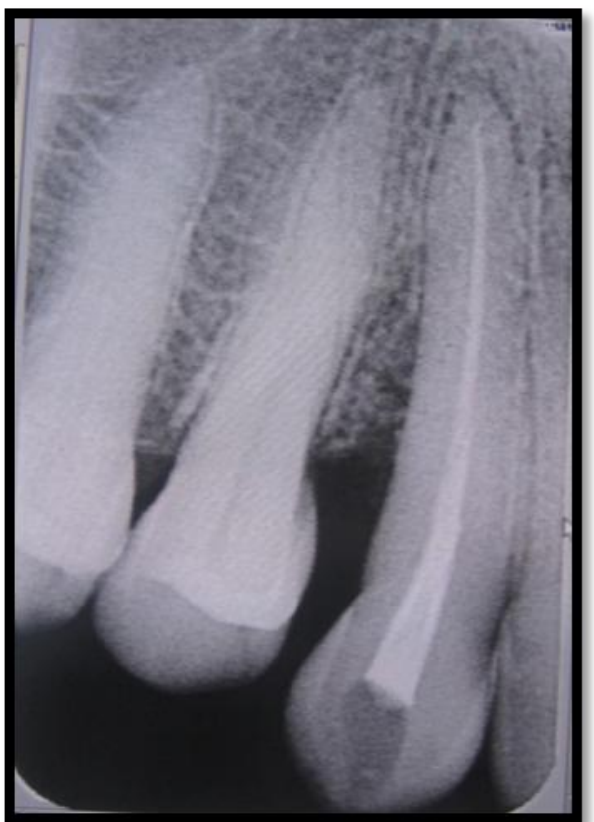

Figure 6(a) : 3 months post operative radiographic view

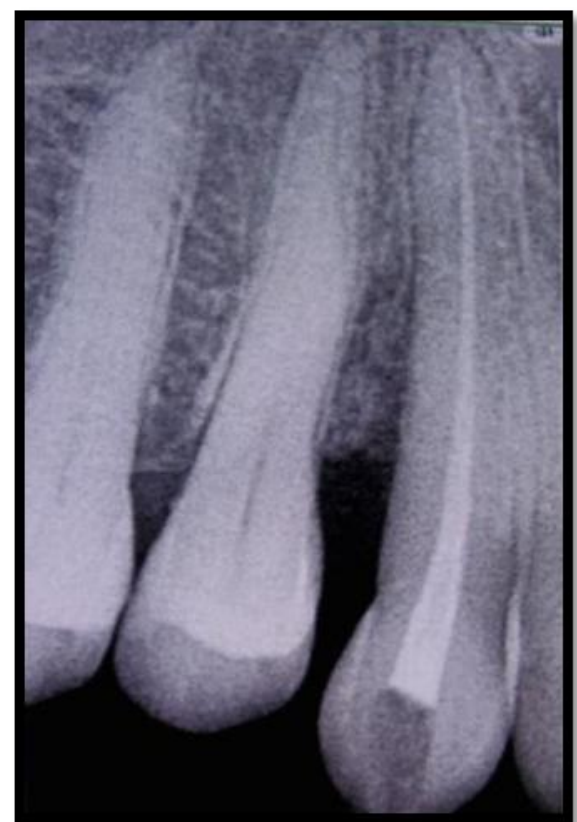

Figure 6(b) : 6 months post operative radiographic view

\section{Discussion:}

The mechanism of periodontal regeneration till date remains a complex and elusive phenomenon. To understand it further, the present case report evaluated the clinical and radiographic efficacy of autologous PRF and bovine derived bone matrix in the treatment of an intrabony defect.

Demineralized bone matrix (DMBM; osseograft ${ }^{\mathrm{TM}}$ ) containing type I collagen obtained from bovine cortical bone samples, is commercially available as nonimmunogenic, resorbable and flowable particles of approximately $250 \mu \mathrm{m}^{23}$. It is deemed to be osteoconductive as well as oseoinductive in nature. The various advantages of DMBM include its ability to act as a space maintainer, easy handling and its cost effectiveness. The only concern regarding DMBM is for its nature of origin i.e. it is obtained from a donor of different species and may result in cross species antigenicity. However, the histological evaluation by Sogal, Tofe et al ${ }^{24}$ confirmed good tolerance and good tissue acceptance of xenografts, with almost completely free of risk of disease transmission.

Platelet rich fibrin, the latest platelet concentrate developed by Choukroun $\boldsymbol{e t ~}^{\boldsymbol{a l}^{21}}$ in 2001 in France, concentrates $97 \%$ of platelets and $>50 \%$ of leukocytes in a specific three dimensional distribution. It consists of intimate assembly of cytokines, glycanic chains and structural glycoproteins enmeshed within a slowly 
polymerized fibrin network. ${ }^{11}$ In an in- vitro comparison of PRF with PRP, He $\boldsymbol{e t} \boldsymbol{a} \boldsymbol{l}^{25}$ demonstrated gradual extended release of autologous growth factors such as PDGF and TGF $\beta$ and better induction of osteoblastic differentiation and proliferation by PRF. The inexpensive nature of PRF, less time consuming and less technique sensitive preparation protocol and favourable molecular properties, makes PRF an obvious choice for endogenous regenerative therapy.

In the present report, a considerable reduction in PPD and CAL gain $(6 \mathrm{~mm}$ each) with minimal recession $(1 \mathrm{~mm})$ was observed 6 months post-operatively. Also, radiographs as early as 3 months, reflected significant bone formation in the periodontal intrabony defect. Almost complete bone fill at 6 months with associated crestal bone formation suggests the arrest of disease activity and its progression. Thus, the results of present report support the role of various growth factors present in the PRF in accelerating the soft and hard tissue healing.

The three walled component of the defect (towards apex), provided the best spatial relationship for bridging of the graft materials with the vascular and cellular elements from the periodontal ligament and adjacent osseous wall. ${ }^{27}$ Also, space maintenance as provided by the defect walls helped to keep the PRF membrane in stable position thus providing protection and retention to the grafted material. ${ }^{27}$ Use of PRF as membrane to cover the apical two walled defect also appeared to be a successful adjunct to the maintenance of defect boundary as minimal recession and good bone fill was observed post-operatively.

Choukroun et al in his histological evaluation on effect of PRF on bone allograft in sinus lift procedure, reported a reduced healing time to 4 months with the addition of PRF. The comparative histological maturation with FDBA alone was observed at 8 months post-operative period only. ${ }^{26}$ Thus, suggesting rapid healing and osteogenic ability of PRF.

The observations of present case report are in accordance with Choukroun's above mentioned study and the recently reported comparative studies involving the combination therapy of PRF with commercially available bone grafts. ${ }^{20,28}$

Simple, easy, fast and cost effective process of PRF preparation without any biochemical involvements hold the major advantage over other derivatives. Also the physiologic functional fibrin matrix has the ability to sustain and progressively release growth factors, cytokines and leukocytes in the surrounding tissues as the matrix degrades over time. All these factors help make Platelet Rich Fibrin the most significant in fibrin technology and endogenous regenerative therapy. ${ }^{23}$

\section{Conclusion}

Among the available grafting modalities, Platelet Rich Fibrin appears to be the most advantageous. Its properties of being the completely natural, physiologic, and economical source of autologous growth factors and cytokines makes it the most sought after treatment option currently available.

From the above report, it can be concluded that the combination of PRF and bone graft can be efficaciously used in the treatment of a periodontal intrabony defects with significantly improved clinical parameters. The use of combination technique also promises additional benefit of rapid and early bone formation. However, clinical trials with larger sample size and confirmatory histological evaluations are required to better assess the clinical benefits of combination approach using PRF with bone grafts.

\section{References}

[1] Lee J, Stavropoulos A, Susin C. Periodontal regeneration: Focus on growth factors and differentiation factors. Dental Clinic of North America. 2010;54:1:93-111

[2] American Academy of Periodontology. Glossary of periodontal terms, 3rd edn. Chicago: American Academy of Periodontology 1992.

[3] Sander L, Karring T. Healing of periodontal lesions in monkeys following the guided tissue regeneration procedure. A histological study. J Clin Periodontol. 1995;22:332-337.

[4] Orsini M, Orsini G, Benlloch D, Aranda JJ, Sanz M. Long-term clinical results on the use of bone replacement grafts in the treatment of intrabony periodontal defects. Comparison of the use of autogenous bone graft plus calcium sulfate to autogenous bone graft covered with a bioabsorbable membrane. J Periodontol. 2008;79:1630.

[5] Cochran DL, Jones A, Heijl L, Mellonig JT, Schoolfield J, King GN. Periodontal regeneration with a combination of enamel matrix proteins and autogenous bone grafting. J Periodontol. 2003;74:1269-8.

[6] Hoidal MJ, Grimard BA, Mills MP, Schoolfield JD, Mellonig JT, Mealey BL. Clinical evaluation of demineralized freeze-dried bone allograft with and without enamel matrix derivative for the treatment of periodontal osseous defects in humans. $J$ Periodontol. 2008;79:2273-80.

[7] Petite $\mathrm{H}$ et al. Tissue engineered bone regeneration. Nat Biotechnol 2000: 18, 959-963

[8] Chen FM, Sheiton RM, Jin Y, Chapple IL. Localized delivery of growth factors for periodontal tissue regeneration: role, stratergies, and perspectives. Med res Rev 2009 29: 472-513

[9] Ross R, Glomset J, Kariya B, Harker L. A platelet-dependent serum factor that stimulates the proliferation of arterial smooth muscle cells in vitro. Proc Natl Acad Sci Usa 1974; 71: 1207-10

[10] Dohan DM, Choukroun J, Diss A, Dohan SL, Dohan AJ, Mouhyi J, Gogly B. Platelet-rich fibrin (PRF): A second-generation platelet concentrate. Part I: Technological concepts and evolution. Oral Surg Oral Med Oral Pathol Oral Radiol Endod. 2006 a ;101:e37-e44 
[11] Dohan DM, Choukroun J, Diss A, et al. Platelet-rich fibrin (PRF): A second-generation platelet concentrate. Part II: Platelet-related biologic features. Oral Surg Oral Med Oral Pathol Oral Radiol Endod. 2006 b;101:e45-e50.

[12] Dohan DM, Choukroun J, Diss A, Dohan SL, Dohan AJ, Mouhyi J, Gogly B. Platelet-rich fibrin (PRF): a second-generation platelet concentrate. Part III: leucocyte activation: a new feature for platelet concentrates? Oral Surg Oral Med Oral Pathol Oral Radiol Endod 2006 c; 101: e51-5.

[13] Sclafani AP. Applications of platelet rich fibrin matrix in facial plastic surgery. Facial Plast Surg 2009;25(4):270-6.

[14] Choukroun J, Diss A, Simonpieri A, Girard MO, Schoeffler C, Dohan SL, Dohan AJ, Mouhyi J, Dohan DM. Platelet-rich fibrin (PRF): a second-generation platelet concentrate. Part V: histologic evaluations of PRF effects on ; bone allograft :maturation in sinus lift. Oral Surg Oral Med Oral Pathol Oral Radiol Endod 2006 b ; 101: 299-303.

[15] Mazor Z, Horowitz RA, Del Corso M, Prasad HS, Rohrer MD, Dohan Ehrenfest DM. Sinus floor augmentation with simultaneous implant placement using Choukroun's platelet rich fibrin as the sole grafting material: a radiologic and histologic study at 6 months. J Periodontol 2009; 80: 2056-64

[16] Pradeep AR, Sharma A. Autologous platelet rich fibrin in the treatment of mandibular Degree II furcation defects: a randomized clinical trial. J Periodontol. 2011;82:1396-1403

[17] Kanakamedala A, Geetha A, Ramakrishnan T, Vijayalakshmi R, Pameela E. Platelet-rich-fibrin: A novel root coverage approach. $J$ Indian Soc Periodontol 2009; 13: 50-54

[18] Pradeep AR, Sharma A. Treatment of 3-Wall Intrabony Defects in Chronic Periodontitis Subjects With Autologous Platelet Rich Fibrin - A Randomized Controlled Clinical Trial. J Periodontol 2011;82:1314-9

[19] Gupta R, Pandit N, Malik R, Sood S. Clinical and radiological evaluation of an osseous xenograft for the treatment of infrabony defects. JCDA 2007;73:513(a-f)

[20] Lekovic V, Camargo Pm, Weinlaender M, Vasilic N. Comparison of platelet rich plasma and bovine porous bone mineral in the treatment of intrabony defects :a re-entry study. J Periodontal 2002;73:198-205

[21] Choukroun J, Adda F, Schoeffler C, Vervelle A. A opportunite' in paroimplantology: the PRF. Implantodontie 2001;42:55-62. (French)

[22] Su CY, Kuo YP, Tseng YH, Su CH, Burnouf T. In vitro release of growth factors from platelet-rich fibrin (PRF): a proposal to optimize the clinical applications of PRF. Oral Surg Oral Med Oral Pathol Oral Radiol Endod 2009; 108(1): 56-61.

[23] Blumenthal N, Sabet T, Barrington E. Healing responses to grafting of combined collagen. J Periodontol. 1986;57:84-94

[24] Sogal, Tofe 1999 Sogal A, Tofe AJ.Risk assessment of bovine spongiform encephalopathy transmission through bone graft material derived from bovine bone used for dental applications. J Periodontal 1999 Sep;70(9):1053-63

[25] He L, Lin Y, Hu X, Zhang Y, Wu H. A comparative study of platelet-rich fibrin (PRF) and plateletrich plasma (PRP) on the effect of proliferation and differentiation of rat osteoblasts in vitro. Oral Surg Oral Med Oral Pathol Oral Radiol Endod. 2009;108(5):707-13.

[26] Choukroun J, Diss A, Simonpieri A, Girard MO, Schoeffler C, Dohan SL, Dohan AJ, Mouhyi J, Dohan DM. Platelet-rich fibrin (PRF): a second-generation platelet concentrate. Part V: histologic evaluations of PRF effects on ; bone allograft :maturation in sinus lift. Oral Surg Oral Med Oral Pathol Oral Radiol Endod 2006 b ; 101: 299-303.

[27] Blumenthal NM, Mario EAF, Salah A, Al-Huwais, Hofbauer AM, Koperski RD. Defect-Determined Regenerative Options for Treating Periodontal Intrabony Defects in Baboons. J Periodontol. 2003;74:10-24.

[28] Pradeep AR, Bajaj P, Rao NS, Aggarwal E. Platelet rich fibrin combined with a porous hydroxyapatite graft for the treatment of 3walled intrabony defects in chronic periodontitis: A Randomized controlled clinical trial. J Periodontal 2012 (ahead of print). 